\title{
The comparison of intrinsic rates of increase among chromosome-substituted lines resistant and susceptible to organophosphate insecticides in Drosophila melanogaster
}

\author{
Takahiro Miyo ${ }^{1 *}$, Yuzuru Oguma ${ }^{2}$, and Brian Charlesworth ${ }^{1}$ \\ ${ }^{1}$ Institute of Cell, Animal and Population Biology, Ashworth Laboratories, University \\ of Edinburgh, King's Buildings, Edinburgh EH9 3JT, UK, and \\ ${ }^{2}$ Institute of Biological Sciences, University of Tsukuba, \\ Ibaraki 305-8572, Japan
}

(Received 9 May 2003, accepted 8 October 2003)

\begin{abstract}
To investigate the genetic basis of the seasonal fluctuations in resistance to three organophosphates, observed within a natural population of Drosophila melanogaster (Meigen), we compared the intrinsic rate of increase, generation time and net reproduction rate among chromosome substitution lines derived from a resistant and a susceptible line, obtained from this natural population. There was significant variation among substituted lines; lines possessing the third chromosome from the resistant line, which confers resistance to the three organophosphates, generally showed lower mean values of these fitness measures. Chromosomal analyses also indicated significant negative contributions of the third chromosome from the resistant line. However, significant positive contributions of the interactions among chromosomes from the resistant line to these fitness measures were also detected. We further conducted a local stability analysis, in which each chromosome-substituted line was assumed to be introduced at a low frequency into the initial susceptible population. It was demonstrated that the resistance factor(s) on the third chromosome tend to decrease in their frequency under both density-independent and juvenile density-regulated conditions. Based on these results, a possible explanation for the seasonal fluctuations in resistance to the three organophosphates observed in the natural population was proposed.
\end{abstract}

Key words: Drosophila melanogaster, insecticide resistance, intrinsic rate of increase, natural populations

\section{INTRODUCTION}

Natural populations of organisms are often age-structured; that is, most natural populations are composed of individuals belonging to various ranges of age-classes, on which fecundities and future survival rates are dependent (Charlesworth, 1994, p. 1). However, populations have usually been treated as homogeneous with respect to age in ecology and population genetics. Research dealing with economically important insect pest populations is no exception to this.

The development of insecticide resistance is an evolutionary process in insect pest populations, in which initially rare genes conferring resistance to insecticides are

Edited by Masa-Toshi Yamamoto

* Corresponding author. E-mail: Takahiro.Miyo@ed.ac.uk selected for and increase in their frequencies in response to insecticide application (Crow, 1957). This phenomenon has been frequently observed in field populations of insect pests (Roush and McKenzie, 1987), and consequently, extensive research on insecticide resistance has been conducted. However, the age-structure of insect pest populations has generally been ignored in the studies of insecticide resistance. Although this kind of simplification has been necessary for understanding the development of insecticide resistance, most real insect pest populations are mixtures of variously aged individuals, whose fecundity and survival rates depend on age. Therefore, any realistic ecological and population genetic models of insecticide resistance should take the age-structure of the populations into account (cf. Charlesworth, 1994, p. 1).

The Katsunuma population of Drosophila melanogaster 
(Meigen) is one of the well-established natural populations in Japan (Hatano et al., 1999). The population size of $D$. melanogaster at Katsunuma increases drastically in the fall, when a large amount of squeezed grapes are dumped outside during the process of wine production (Watanabe and Oshima, 1970; Miyo et al., 2000). In this Katsunuma population of $D$. melanogaster, seasonal fluctuations in susceptibility to three organophosphate insecticides were observed, in which resistance levels of the three organophosphates tended to decrease in the fall (Miyo et al., 2000). Evaluation of resistance to the three organophosphates, and productivity (a major fitness component), of each isofemale line established from the same Katsunuma population demonstrated the existence of negative correlations between productivity and resistance to the three organophosphates (Miyo and Oguma, 2002). After constructing chromosome-substituted lines between a resistant and a susceptible line established from the same Katsunuma population, the genetic basis of the negative correlations was examined (Miyo and Oguma, 2002). Positive effects on resistance to the three organophosphates and a negative effect on productivity were detected for the third chromosome of the resistant line. Based on the genetic analysis of the negative correlations between organophosphate resistance and productivity, fitness disadvantages of resistant genotypes were suggested, which could explain the seasonal fluctuations in resistance to the three organophosphates observed in the Katsunuma population of D. melanogaster (Miyo and Oguma, 2002).

Although the negative correlations were demonstrated between resistance to the three organophosphates and productivity, it is possible that other fitness components compensate for the effect of productivity on the fitness of the resistant line. Since all processes contributing to reproduction affect fitness measures (Anderson and Watanabe, 1974), conclusions derived from analyses based on one of the fitness components might be wrong (Caswell, 2001, p. 280). The Katsunuma population of D. melanogaster in the fall may be treated as a densityindependent population created by sudden appearance of a large amount of squeezed grapes dumped outside during the process of wine production (Miyo et al., 2000).

In this study, we compared the intrinsic rate of increase, which represents fitness under density-independent conditions (Charlesworth, 1994, Chapter 4), among chromosome substitutions between lines resistant and susceptible to the three organophosphates. To estimate the intrinsic rate of increase of each chromosome-substituted line, age-specific fecundity and survival rates were collected for individual adult flies of each line. In order to evaluate the fate of the resistance gene(s), increase or decrease, within the initial susceptible population, we further conducted a local stability analysis, in which each chromosome-substituted line was assumed to be intro- duced at a low frequency into the initial susceptible population, whose density is either not regulated or is regulated by juvenile mortality. Based on these analyses, the dynamics of genetic variation in resistance to the three organophosphates in the Katsunuma population of D. melanogaster were analyzed.

\section{MATERIALS AND METHODS}

Drosophila lines. To compare intrinsic rates of increase among genotypes resistant and susceptible to organophosphate insecticides, we obtained demographic data from chromosome-substituted lines between a resistant inbred line \#609-10 and a susceptible inbred line \#451-4, constructed by using a balancer stock $w$; Sp/SM1; Pr Dr/ TM3 (Miyo et al., 2002; Miyo and Oguma, 2002). After more than 15 generations of continual sib mating, both of the inbred lines were established from respective isofemale lines, which were derived from the same natural population collected at Katsunuma on July 31, 1997 (Miyo et al., 2001). Because at this time the Katsunuma population of $D$. melanogaster had not yet started expanding, we chose to use these lines for the genetic analysis. In the fall, the Katsunuma population starts growing on masses of squeezed grapes dumped in vineyards (Watanabe and Oshima, 1970; Miyo et al., 2000). Line \#609-10 had low-to-moderate, but significant levels of resistance to three organophosphates, malathion, prothiophos and fenitrothion (Miyo et al., 2001). Based on regression analyses, the major resistance factor(s) for the three organophosphates was mapped to the third chromosome (Miyo and Oguma, 2002).

Flies used for experiments were grown on glucoseyeast-cornmeal-agar medium in glass vials $(3 \mathrm{~cm}$ in diameter and $10.5 \mathrm{~cm}$ in height) in incubators at $25 \pm 0.5^{\circ} \mathrm{C}$ with a photoperiod of 14: 10 (L: D) h.

In the following, each chromosome-substituted line was expressed by its chromosome composition. For example, RSR indicates the line possessing the $\mathrm{X}$ and third chromosomes derived from the resistant line \#609-10 and the second chromosome from the susceptible line \#451-4.

Age-specific survivorship and fecundity. The intrinsic rate of increase for each chromosome-substituted line was estimated, based on life history data collected for each individual adult fly (cf. Lenski and Service, 1982; McGraw and Caswell, 1996). One pair of adult flies that emerged within six hours was placed in a food vial $(3 \mathrm{~cm}$ in diameter and $10.5 \mathrm{~cm}$ in height) and transferred into a new vial every day. When the flies were transferred, it was checked whether the flies were alive or dead, and newly emerged adult flies were counted every day. 16 pairs were prepared for each chromosome-substituted line.

To estimate intrinsic rates of increase, the demographic 
dominance of females was assumed (Charlesworth, 1994, p. 4). Half of the newly emerged adult flies on each day were considered in the estimates of age-specific fecundity in this study. Age-specific survival probability was assigned to be one when the transferred adult female was alive and zero when she was dead (Lenski and Service, 1982; McGraw and Caswell, 1996). Because adult females were transferred, the age-specific survival rate from egg to pupal stages was one. Ages of the flies placed in the food vials were estimated as the duration from the entry of the adult flies to first emergence of their offspring.

The survival function, $l(x)$, and the fecundity function, $m(x)$, were determined, based on age-specific survival probabilities and fecundity over 19 days in this study. The Katsunuma population starts expansion from late September to early October, when a large amount of squeezed grapes were dumped outside in the process of wine production. However, the population rapidly decreased in mid-November, probably because the dumped grapes had dried and because it got colder. In fact, no $D$. melanogaster flies were collected in mid-November (author's observation on November 15, 2000). Therefore, the explosion of the Katsunuma population took place during the short period of time, and age-specific fecundity and survival probabilities for 19 days would be sufficient for calculating the intrinsic rate of increase.

Estimation of intrinsic rates of increase. The intrinsic rates of increase, $r$, which measure the rate of population growth per unit time, were calculated numerically by solving the Euler-Lotka equation for the discrete ageclass model

$$
\Sigma \mathrm{e}^{-r x} l(x) m(x)=1
$$

(Charlesworth, 1994, equation (1.45)) by Newton-Raphson iteration:

$$
r_{n}=r_{n-1}+\left(\Sigma \mathrm{e}^{-r_{n-1} x} l(x) m(x)-1\right) / \Sigma x \mathrm{e}^{-r_{n-1} x} l(x) m(x)(\mathrm{n}=1,2 \ldots)
$$

where $n$ represents the number of the iteration.

In the Newton-Raphson iteration, initial values of the intrinsic rates of increase, $r_{0}$, were estimated by

$$
r_{0}=(\Sigma l(x) m(x)-1) / \Sigma x l(x) m(x)
$$

(Charlesworth, 1994, equation (1.46)). At the same time, generation time, $T=\Sigma x \mathrm{e}^{-r x} l(x) m(x)$ in equation (1), which is the mean age of mothers in a population growing at a given rate $r$, (Charlesworth, 1994, equation (1.47c)), and the net reproduction rate, $R_{0}=\Sigma l(x) m(x)$ in equation (2), measuring the expectation of total female offspring to a female at birth (Charlesworth, 1994, p. 29), were also estimated by Newton-Raphson iteration. Newton-Raphson iteration was performed using a FORTRAN 77 program.

Local stability analysis. We conducted a local stability analysis to examine the probability of survival of variants with the characteristics of each chromosomesubstituted line, when introduced into the susceptible SSS population (cf. Charlesworth, 1972; Charlesworth, 1994, §4.3). To do this, we first compared the difference in the intrinsic rate of increase between SSS and each of the other substituted lines. The differences in the intrinsic rate of increase between two homozygous lines determine the direction of change in frequency of a rare recessive gene, introduced into the density-independent homozygous population (Charlesworth, 1994, p. 153). The intrinsic rate of increase of heterozygous lines cannot be determined in this study, because heterozygous lines cannot be maintained pure in our fecundity assay condition (Charlesworth, 1993). However, deleterious mutations are mostly partially recessive (Hughes, 1995; Houle et al. 1997; Charlesworth and Hughes, 2000). Therefore, the differences in the intrinsic rate of increase between two homozygous lines provide some indications of the fate of the rare resistance genes.

Although the Katsunuma population in the fall is considered to be density-independent, we further tested a larval density regulated situation, which might be more realistic for a local scale of the Katsunuma population. This situation was examined, by calculating the intrinsic rate of increase of each chromosome-substituted line, in which the juvenile density regulating factors were kept constant at the level, which provides the susceptible SSS population with a zero rate of population growth (cf. Charlesworth, 1993).

Each chromosome-substituted line was assumed to be introduced at a low frequency into an initial population, whose density is regulated in response to the mean net reproduction rate of line SSS (72.9; Fig. 1), and exposed to the same juvenile mortality 0.0137 (1/72.9), which should provide the susceptible SSS population with a zero rate of population growth (cf. Charlesworth, 1972). Under this assumption, the intrinsic rate of increase was calculated for each chromosome-substituted line. If an introduced line has a higher intrinsic rate of increase than line SSS, the intrinsic rate of increase of the line under this condition would be positive, suggesting the spread of the line into the susceptible population. If lower, it would be negative, suggesting the exclusion of the line from the susceptible population.

Statistical procedures. To compare the intrinsic rate of increase, generation time and net reproduction rate, among chromosome-substituted lines, two statistical analyses were conducted. Because the intrinsic rate of 
(a) Intrinsic rate of increase

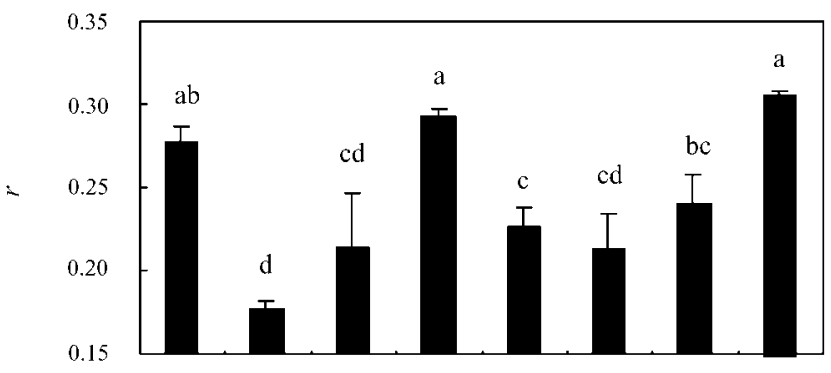

(b) Gencration time

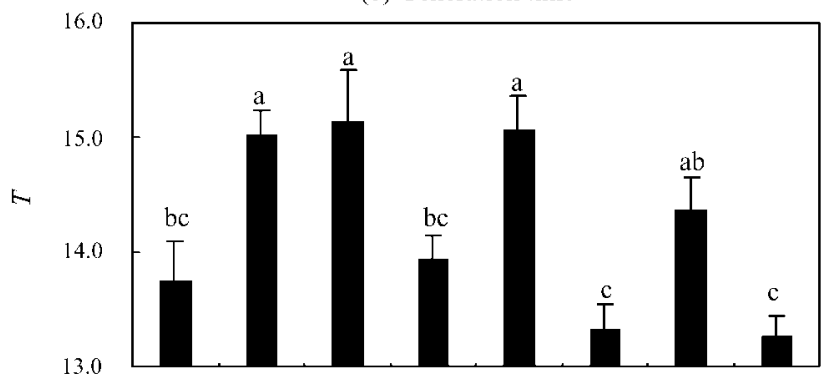

(c) Net reproduction rate

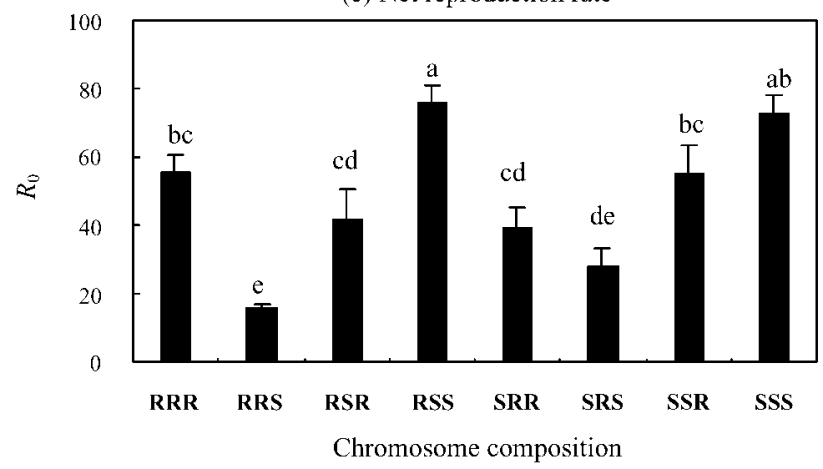

Fig. 1. Mean values of the intrinsic rate of increase (a), generation time (b), and net reproduction rate (c) for homozygous combinations of chromosome-substituted lines between a resistant and a susceptible line. Each chromosome-substituted line was indicated by its chromosome composition (X, second, and third chromosome in order). $\mathrm{R}$ and $\mathrm{S}$ indicate the origin of the chromosome: $\mathrm{R}$ for the resistant line \#609-10 and $\mathrm{S}$ for the susceptible line \#451-4. The error bars indicate the standard errors of the means. Bars followed by the same letters are not significantly different (Duncan's multiple range test, $P>0.05$ ).

increase cannot be defined for the females that produced no offspring, we used two methods, which differ in treating these females ( 16 out of the 128 adult females did not produce adult offspring).

For the first method, estimates of intrinsic rates were calculated for each adult female from each line and subjected to analysis of variance (PROC GLM; SAS Institute, 1985), in which the females that produced no offspring were treated as missing values. Duncan's multiple range tests were conducted for comparing the mean values of the intrinsic rate of increase, generation time and net reproduction rate, among chromosome-substituted lines $(P=0.05$; SAS Institute, 1985).

The other method was the jackknife (cf. Lenski and Service, 1982; Houle et al, 1997). The 15 or 16 pseudovalues of the intrinsic rate of increase, generation time and net reproduction rate were calculated for each line by excluding the data for one female, and determining $l(x)$ and $m(x)$ for the cohort of remaining females. This step was repeated for each female of a line. Although the ages of the adult females that produced no adult offspring cannot be assigned in this study, 14 out of the 16 adult females that produced no offspring survived throughout the 19day census period; therefore, the age-specific survival rates $(l(x))$ for these females were assigned to be one. Two females, one RRS and one RSR female, which did not produce any adult offspring and died during the 19-day census period, were excluded from the jackknife procedure, because the age-specific survival rates for these females cannot be defined. The mean values of the intrinsic rate of increase, generation time and net reproduction rate were compared among chromosome-substituted lines, based on the $95 \%$ confidence intervals.

To evaluate the effects of each of the three major chromosomes of the resistant line and their interactions on the intrinsic rate of increase, generation time and net reproduction rate, multiple regression analyses were conducted (PROC GENMOD; SAS Institute, 1985; Manly, 1985; Johnston, 2000). Explanatory variables were assigned to be one if the chromosome was derived from the resistant line (R) and zero if it was from the susceptible line (S) (Johnston, 2000; Miyo and Oguma, 2002). Goodness-of -fit of the model was assessed, based on Pearson $\chi^{2}$ values.

In the local stability analysis, the differences in the intrinsic rate of increase between each of the chromosome-substituted lines and line SSS were tested by using the $t$-test assuming unequal variances (PROC TTEST; SAS Institute, 1985). Because the mean intrinsic rate of increase of line SSS was the highest among the chromosome-substituted lines, we tested the one-sided alternative hypothesis that the difference in the mean intrinsic rate of increase between two chromosome-substituted lines was significantly negative.

To test the one-sided alternative hypothesis that the mean intrinsic rate of increase under the juvenile densitydependent condition was significantly negative, a onesample $t$-test was conducted for each chromosome-substituted line (PROC TTEST; SAS Institute, 1985). Because the intrinsic rate of increase under this local stability condition cannot be defined for females that produced less than one offspring, a total of 18 females were treated as missing values. The jackknife procedure was also implemented, in which the two females that produced no offspring and died during the 19-day census period were excluded for the same reason as mentioned above. 


\section{RESULTS}

Variation among chromosome-substituted lines.

Results of analysis of variance of the intrinsic rate of increase, generation time and net reproduction rate are shown in Table 1. Significant variation was detected among chromosome-substituted lines in the intrinsic rate of increase, generation time, and net reproduction rate. Results of the Duncan's multiple range tests for the mean values are shown in Fig. 1 . In the comparison of the mean values of the intrinsic rate of increase $(r)$, lines possessing the second and/or third chromosomes from the resistant line exhibited significantly lower intrinsic rates (Fig. 1-a). However, the mean intrinsic rate of increase of line RRR, possessing all of the three major chromosomes from the resistant line \#609-10, was not significantly different from that of line SSS, possessing all of the major chromosomes from the susceptible line \#451-4. In case of generation time $(T)$, lines possessing the third chromosome from the resistant line generally had a longer generation time; on the other hand, generation time tended to be shorter in the lines possessing the third chromosome from the susceptible line (Fig. 1b). However, this tendency was reversed in lines RRR and RRS; the generation time of line RRR was not significantly different from that of line SSS, and a significant difference was detected between lines RRS and SSS. In the comparison of the mean values of the net reproduction rate $\left(R_{0}\right)$, the pattern was similar to the case of the intrinsic rate of increase (Fig. 1-c). However, the significance of the differences was less clear. In general, lines possessing the second chromosome from the resistant line showed significantly lower net reproduction rates; lines possessing the third chromosome from the resistant line also tended to have lower net reproduction rates. However, line RRR had a net reproduction rate, which was not significantly different from that of line SSS.

In order to take the females that produced no offspring into account, not only did we conduct the analysis of variance, but we also implemented the jackknife, in which the females producing no offspring were involved. Results of the jackknife are listed in Table 2 , with mean values and their $95 \%$ confidence intervals. The results of the jackknife and analysis of variance were generally similar. In comparison of the intrinsic rate of increase $(r)$, lines having the second and/or third chromosomes from the resistant line had significantly lower intrinsic rates, based on 95\% confidence intervals, although the difference between lines RRR and SSS was not significant. In case of generation time $(T)$, lines having the third chromosome from the resistant line generally showed a significantly longer generation time, although this relationship was reversed in lines RRR and RRS. In case of the net reproduction rate $\left(R_{0}\right)$, lines possessing the second and/or third chromosomes from the resistant line tended to have lower net reproduction rates than that of line SSS. However, the differences were less clear, based on $95 \%$ confidence intervals. Summing up these jackknife results, it could be suggested that treating the data for the females that produced no offspring as missing values did not have a

Table 1. Analysis of variance of the intrinsic rate of increase $(r)$, generation time $(T)$, and net reproduction rate $\left(R_{0}\right)$.

\begin{tabular}{|c|c|c|c|c|c|c|c|c|c|}
\hline \multirow{2}{*}{$\begin{array}{l}\text { Source of } \\
\text { Variation }\end{array}$} & \multicolumn{3}{|c|}{$r$} & \multicolumn{3}{|c|}{$T$} & \multicolumn{3}{|c|}{$R_{0}$} \\
\hline & d. f. & MS & $\mathrm{F}$ & d. f. & MS & $\mathrm{F}$ & d. f. & MS & $\mathrm{F}$ \\
\hline Line & 7 & 0.028 & $8.48^{* * *}$ & 7 & 8.060 & $7.48^{* * *}$ & 7 & 6179.983 & $12.18^{* * * *}$ \\
\hline Error & 104 & 0.003 & & 104 & 1.077 & & 104 & 507.476 & \\
\hline
\end{tabular}

*** $P<0.0001$.

Table 2. The mean intrinsic rate of increase $(r)$, generation time $(T)$, net reproduction rate $\left(R_{0}\right)$, intrinsic rate of increase under juvenile density-regulated condition $\left(r_{\text {density }}\right)$, and their $95 \%$ confidence intervals for each chromosome-substituted line, estimated by the jackknife.

\begin{tabular}{llllll}
\hline \hline Line & $\mathrm{n}$ & \multicolumn{1}{c}{$r \pm 95 \% \mathrm{CI}$} & \multicolumn{1}{c}{$T \pm 95 \% \mathrm{CI}$} & $R_{\mathbf{o}} \pm 95 \%$ CI & $r_{\text {density }} \pm 95 \%$ CI \\
\hline RRR & 16 & $0.2796 \pm 0.0214 \mathrm{abc}$ & $13.37 \pm 0.36 \mathrm{ab}$ & $52.00 \pm 12.78 \mathrm{ab}$ & $-0.0221 \pm 0.0159$ \\
$\mathrm{RRS}$ & 15 & $0.1696 \pm 0.0185 \mathrm{~d}$ & $15.03 \pm 0.51 \mathrm{e}$ & $13.57 \pm 3.89 \mathrm{~d}$ & $-0.1042 \pm 0.0184$ \\
$\mathrm{RSR}$ & 15 & $0.2150 \pm 0.0519 \mathrm{~cd}$ & $14.58 \pm 0.94 \mathrm{bcde}$ & $24.97 \pm 16.27 \mathrm{bcd}$ & $-0.0645 \pm 0.0403$ \\
$\mathrm{RSS}$ & 16 & $0.2898 \pm 0.0158 \mathrm{ab}$ & $13.82 \pm 0.39 \mathrm{abcd}$ & $71.12 \pm 14.30 \mathrm{a}$ & $-0.0014 \pm 0.0127$ \\
$\mathrm{SRR}$ & 16 & $0.2357 \pm 0.0265 \mathrm{c}$ & $14.68 \pm 0.79 \mathrm{cde}$ & $36.69 \pm 12.98 \mathrm{bc}$ & $-0.0419 \pm 0.0222$ \\
SRS & 16 & $0.2272 \pm 0.0315 \mathrm{c}$ & $13.52 \pm 0.50 \mathrm{abd}$ & $24.41 \pm 11.18 \mathrm{~cd}$ & $-0.0709 \pm 0.0324$ \\
SSR & 16 & $0.2637 \pm 0.0230 \mathrm{bc}$ & $14.59 \pm 0.46 \mathrm{ce}$ & $54.97 \pm 17.87 \mathrm{ab}$ & $-0.0171 \pm 0.0204$ \\
SSS & 16 & $0.3008 \pm 0.0131 \mathrm{a}$ & $13.24 \pm 0.39 \mathrm{a}$ & $68.38 \pm 14.39 \mathrm{a}$ & $-0.0040 \pm 0.0140$ \\
\hline
\end{tabular}

Each chromosome-substituted line was indicated by its chromosome composition (X, second, and third chromosome in order). Mean values followed by the same letter were not significantly different, based on the overlap of $95 \%$ confidence intervals. 
large effect on the results of this study.

Chromosomal analysis. Results of multiple regression analyses of chromosomal effects on the intrinsic rate of increase, generation time, and intrinsic rate of increase under the juvenile density-dependent condition are listed in Table 3. Significant negative contributions to the intrinsic rate of increase $(r)$ was detected from both the second and the third chromosome from the resistant line, and significant positive effects were detected from the interaction between the second and the third chromosome from the resistant line, and from the interaction among the $\mathrm{X}$, second and third chromosomes from the resistant line. Pearson $\chi^{2}$ values indicated that the model fitted the data well $\left(\chi^{2}=0.3381, \mathrm{~d}\right.$. f. $\left.=104, P>0.995\right)$. In the case of generation time $(T)$, a significant negative contri- bution was detected only from the third chromosome, and a significant positive effect was detected from the interaction among the $\mathrm{X}$, second and third chromosomes. The model fitted the data adequately $\left(\chi^{2}=112.04\right.$, d. f. $=104$, $P=0.278$ ). In case of the intrinsic rate of increase under the density-dependent condition regulated by juvenile mortality of line SSS $\left(r_{\text {density }}\right)$, a significant negative contribution was detected from both the second and the third chromosomes from the resistant line. However, a significant positive effect was detected only from the interaction between the second and the third chromosome from the resistant line. The model fitted the data well $\left(\chi^{2}=\right.$ 0.2167 , d. f. $=102, P>0.995)$. In case of the net reproduction rate, the model was not adequate $\left(\chi^{2}=\right.$ 52777.5382, d. f. $=104, P<0.0001)$; therefore, parameter estimates were not listed for the net reproduction rate.

Table 3. Maximum likelihood estimation of the effects of the three major chromosomes from the resistant line and their interactions on the intrinsic rate of increase $(r)$, generation time $(T)$, and the intrinsic rate of increase under the juvenile density-dependent condition $\left(r_{\text {density }}\right)$.

\begin{tabular}{|c|c|c|c|c|c|c|}
\hline Trait & Parameter & d. f. & Estimate & $\mathrm{SE}$ & $\chi^{2}$ & $P$ \\
\hline \multirow[t]{8}{*}{$r$} & $\beta_{0}$ & 1 & 0.3042 & 0.0142 & 459.91 & $<0.0001$ \\
\hline & $\beta_{\mathrm{X}}$ & 1 & -0.0128 & 0.0201 & 0.41 & 0.5243 \\
\hline & $\beta_{\mathrm{II}}$ & 1 & -0.0926 & 0.0204 & 20.58 & $<0.0001$ \\
\hline & $\beta_{\mathrm{III}}$ & $\mathbf{1}$ & -0.0653 & 0.0197 & 10.93 & 0.0009 \\
\hline & $\beta_{\mathrm{X}-\mathrm{II}}$ & 1 & -0.0228 & 0.0292 & 0.61 & 0.4342 \\
\hline & $\beta_{\mathrm{X}-\mathrm{III}}$ & 1 & -0.0136 & 0.0304 & 0.20 & 0.6560 \\
\hline & $\beta_{\text {IIIIII }}$ & 1 & 0.0787 & 0.0284 & 7.68 & 0.0056 \\
\hline & $\beta_{\mathrm{X}-\mathrm{II}-\mathrm{III}}$ & 1 & 0.1002 & 0.0422 & 5.65 & 0.0175 \\
\hline \multirow[t]{8}{*}{$T$} & $\beta_{0}$ & 1 & 13.2602 & 0.2582 & 2636.55 & $<0.0001$ \\
\hline & $\beta_{\mathrm{X}}$ & 1 & 0.6748 & 0.3652 & 3.41 & 0.0647 \\
\hline & $\beta_{\text {II }}$ & 1 & 0.0547 & 0.3717 & 0.02 & 0.8831 \\
\hline & $\beta_{\mathrm{III}}$ & 1 & 1.1036 & 0.3595 & 9.43 & 0.0021 \\
\hline & $\beta_{\mathrm{X}-\mathrm{II}}$ & 1 & 1.0250 & 0.5308 & 3.73 & 0.0535 \\
\hline & $\beta_{\mathrm{X}-\mathrm{III}}$ & 1 & 0.0986 & 0.5541 & 0.03 & 0.8588 \\
\hline & $\beta_{\text {IIIIII }}$ & 1 & 0.6434 & 0.5171 & 1.55 & 0.2134 \\
\hline & $\beta_{\mathrm{X}-\mathrm{II}-\mathrm{III}}$ & 1 & -3.1161 & 0.7674 & 16.49 & $<0.0001$ \\
\hline \multirow[t]{8}{*}{$r_{\text {density }}$} & $\beta_{0}$ & 1 & -0.0045 & 0.0115 & 0.15 & 0.6960 \\
\hline & $\beta_{\mathrm{X}}$ & 1 & 0.0048 & 0.0162 & 0.09 & 0.7674 \\
\hline & $\beta_{\mathrm{II}}$ & 1 & -0.0676 & 0.0168 & 16.15 & $<0.0001$ \\
\hline & $\beta_{\mathrm{III}}$ & 1 & -0.0470 & 0.0160 & 8.68 & 0.0032 \\
\hline & $\beta_{\mathrm{X}-\mathrm{II}}$ & 1 & -0.0337 & 0.0238 & 2.00 & 0.1571 \\
\hline & $\beta_{\mathrm{X}-\mathrm{III}}$ & 1 & 0.0131 & 0.0251 & 0.27 & 0.6030 \\
\hline & $\beta_{\text {II-III }}$ & 1 & 0.0683 & 0.0232 & 8.67 & 0.0032 \\
\hline & $\beta_{\mathrm{X}-\mathrm{II}-\mathrm{III}}$ & 1 & 0.0438 & 0.0346 & 1.60 & 0.2054 \\
\hline
\end{tabular}

Boldface indicates significant parameter estimates $(P<0.05)$. 
Table 4. Differences in the intrinsic rate of increase under the density-independent condition between SSS and each of the other chromosome-substituted lines.

\begin{tabular}{clllllll}
\hline \hline & $r_{\mathrm{RRR}}-r_{\mathrm{SSS}}$ & $r_{\mathrm{RRS}}-r_{\mathrm{SSS}}$ & $r_{\mathrm{RSR}}-r_{\mathrm{SSS}}$ & $r_{\mathrm{RSS}}-r_{\mathrm{SSS}}$ & $r_{\mathrm{SRR}}-r_{\mathrm{SSS}}$ & $r_{\mathrm{SRS}}-r_{\mathrm{SSS}}$ & $r_{\mathrm{SSR}}-r_{\mathrm{SSS}}$ \\
\hline Difference & -0.028 & -0.128 & -0.092 & -0.013 & -0.079 & -0.093 & -0.065 \\
$t$ value & -2.50 & -18.69 & -2.67 & -1.82 & -5.78 & -4.02 & -3.39 \\
d. f. & 17.6 & 21.4 & 8.2 & 24 & 16.3 & 13.7 & 16.2 \\
$P$ & 0.0113 & $<0.0001$ & 0.0138 & 0.0405 & $<0.0001$ & 0.0007 & 0.0018 \\
\hline
\end{tabular}

Each chromosome-substituted line was indicated by its chromosome composition (X, second, and third chromosome in order).

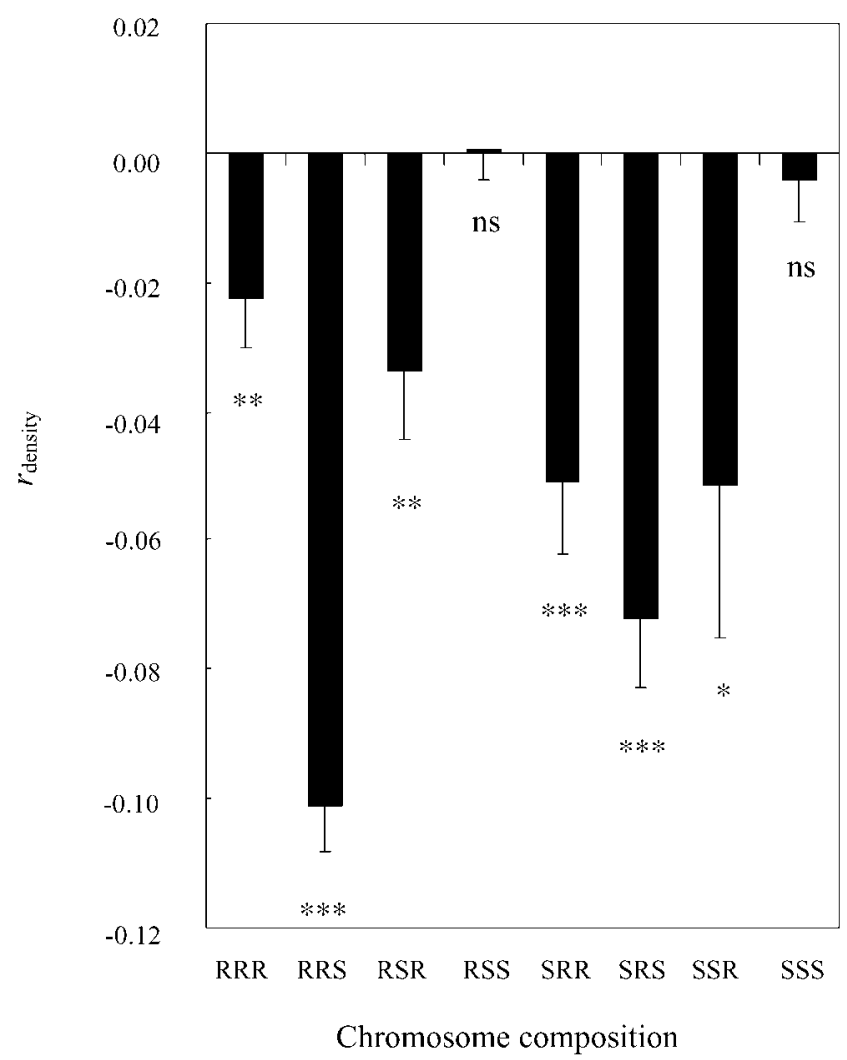

Fig. 2. Mean values of the intrinsic rate of increase under the juvenile density-dependent condition, assumed to be regulated by the mean net reproduction rate of line SSS for homozygous combinations of chromosome-substituted lines. Each line was indicated by its chromosome composition (X, second, and third chromosome in order). The error bars indicate the standard errors of the means. Asterisks indicate the significance levels. ns, not significant; * $P<0.05$; ** $P<0.01$; *** $P<0.001$.

Local stability analysis. The differences in the mean intrinsic rate of increase between each of the chromosome-substituted lines and SSS are listed in Table 4. In all cases, the differences, $r_{\mathrm{ijk}}-r_{\mathrm{SSS}}(\mathrm{i}, \mathrm{j}, \mathrm{k}=\mathrm{R}$ or $\mathrm{S})$, were significantly negative (one-sided $P<0.05$ ).

The mean intrinsic rates of increase under the densitydependent condition regulated by juvenile mortality of line SSS are displayed in Fig. 2. All lines, except lines RSS and SSS, exhibited significantly negative intrinsic rates of increase (one-sided $P<0.05$ ). The mean values of the intrinsic rate of increase of lines RSS and SSS were not significantly different from zero. The jackknife also showed significantly negative intrinsic rates of increase under the density-regulated condition in these chromosome-substituted lines, based on 95\% confidence intervals, although that of line SSR was not significantly different from zero in this case (Table 2).

\section{DISCUSSION}

In the previous study, we demonstrated negative correlations between resistance to the three organophosphates and productivity in the Katsunuma population of D. melanogaster, in which the third chromosome of the resistant line \#609-10 could provide a genetic basis of the negative correlations at least in part (Miyo and Oguma, 2002). However, all processes in reproduction could affect fitness measures (Anderson and Watanabe, 1974). Therefore, the effect of the third chromosome of the same resistant line on fitness itself was necessary to be measured. In many cases, the Darwinian fitness of a genotype can be measured by the intrinsic rate of increase (Charlesworth, 1993).

In this study, we further compared the intrinsic rate of increase, generation time and net reproduction rate, among the same set of chromosome substitutions between the resistant and susceptible lines as in the previous study, in order to reveal the genetic basis of the seasonal fluctuations in resistance to the three organophosphates, observed within the Katsunuma population of $D$. melanogaster. The resistant and susceptible lines were obtained from the Katsunuma population on July 31, 1997, when the Katsunuma population had not expanded yet (Miyo et al. 2000). There was significant variation among chromosome-substituted lines (Table 1), and the lines possessing the third chromosome from the resistant line, which were revealed to be resistant genotypes (Miyo and Oguma, 2002), generally exhibited lower fitness (Fig. 1; Table 2). Indeed, the third chromosome from the resistant line, on which the major resistance factor(s) were located, contributed negatively to these fitness measures (Table 3). These results suggest that resistance factor(s) on the third chromosome could have disadvantageous effects on fitness. 
If a rare, non-recessive allele $A_{2}$ is introduced into a density-independent initial $A_{1} A_{1}$ population, the change in the frequency of allele $\mathrm{A}_{2}, p_{2}$, is approximated as

$$
\Delta \ln p_{2} \approx\left(r_{12}-r_{11}\right)
$$

(Charlesworth, 1994, equation (4.30)). When $r_{22}<r_{12}<$ $r_{11}$, the condition $p_{1}=1$ is stable, and $p_{2}=1$ is unstable, suggesting that a rare allele $\mathrm{A}_{2}$ is excluded from the $\mathrm{A}_{1} \mathrm{~A}_{1}$ population (Charlesworth, 1994, p. 151). On the other hand, if a rare, recessive allele $A_{2}$ was introduced, the frequency of $A_{2}$ changes approximately as

$$
\Delta p_{2} \approx\left(p_{2}\right)^{2}\left(r_{22}-r_{11}\right)
$$

(Charlesworth, 1994, equation (4.34)). A rare, recessive allele $\mathrm{A}_{2}$ can invade the $\mathrm{A}_{1} \mathrm{~A}_{1}$ population only when $r_{22}>$ $r_{11}$ (Charlesworth, 1994, p. 152). This study demonstrated that the difference in the intrinsic rate of increase between each of the homozygous chromosome-substituted lines and line SSS was significantly negative (Table 4), suggesting that resistance factor(s) on the third chromosome tend to be excluded from the initial susceptible SSS population under the density-independent condition, if the dominance levels of chromosomes from the resistant line are within the range from dominant to recessive.

In order to estimate the intrinsic rate of increase of heterozygous combinations of chromosome-substituted lines, our fecundity assay method has to make simplifying assumptions, because we cannot maintain pure heterozygous lines. We have neglected the dominance levels of the resistant chromosomes, and simply tested the difference in the intrinsic rate of increase between two homozygous combinations of chromosome-substituted lines. However, most deleterious mutations are partially recessive (Hughes, 1995; Houle et al., 1997; Charlesworth and Hughes, 2000). In addition, mutations with heterozygote superiority are rare (Simmons and Crow, 1977; Houle et al., 1997). Under heterozygote superiority, alleles could be maintained at intermediate frequencies within equilibrium populations (Charlesworth and Hughes, 2000). If resistance genes show heterozygous superiority for fitness, the frequencies of the resistance genes must have been maintained at intermediate frequencies within insect populations, even before insecticides were introduced. This hypothesis could be rejected by the obvious fact that insecticides had been very effective during the initial period when insecticides were introduced for controlling insect pest populations, which indicates that resistance genes had been quite rare within initial insect populations (Crow, 1957). Therefore, our suggestion that resistance factor(s) on the third chromosome tend to be excluded from the SSS population could be reasonable.

In order to make the results solid, a precise estimation of the intrinsic rate of increase of heterozygous combina- tions of chromosome-substituted lines should be performed. This estimation is also necessary from the standpoint of the origin of resistance genes, which is still obscure (Daborn et al., 2002), because most rare mutant alleles would be in heterozygous conditions within initial large natural populations (Simmons and Crow, 1977). One way to tackle this problem might involve population cage experiments, in which each chromosome is allowed to compete against the third chromosome balancer, such as TM3 (cf. Houle et al. 1992).

Although the Katsunuma population of D. melanogaster is considered to be density-independent in the fall, there could be severe competition for larval survival in natural populations of Drosophila (Simmons and Crow, 1977). This could be true especially at the local scale, such as within one drop of grapes. In this study, we calculated the intrinsic rate of increase for each chromosome-substituted line, in which each line was assumed to be introduced at a rare frequency into the initial susceptible population, whose density is regulated by the mean net reproduction rate of line SSS, and exposed to the same juvenile mortality. This juvenile mortality should provide the susceptible SSS population with a zero rate of population growth. The intrinsic rates of increase of resistant genotypes (RRR, RSR, SRR and SSR) under this juvenile-density regulated condition were all negative (Fig. 2), suggesting that the resistance factor(s) on the third chromosome tend to decrease in frequency, even under this larval density-regulated condition.

These results suggest that the frequency of the resistance factor(s) on the third chromosome from the resistant line tend to decrease under insecticide-free environments or after insecticide application is ceased. This could explain the seasonal fluctuations in resistance to the three organophosphates, observed within this Katsunuma population at least in part. Variation in the intrinsic rate of increase among the resistant genotypes could trigger the change of the genetic constitution within the fall population, in which the relative frequencies of the resistant genotypes are reduced. Consequently, the levels of resistance to the three organophosphates could decrease in the fall population. Because the resistance factor(s) on the third chromosome confer resistance to all of the three organophosphates (Miyo and Oguma, 2002), the decrease in the frequency of the resistance factor(s) could result in the simultaneous decrease in resistance levels for all of the three organophosphates, as observed in the Katsunuma population (Miyo et al., 2000).

The disadvantageous effects of resistance genes on fitness or fitness components have been controversial because field observation and experimental evidence have been limited (Chevillon et al., 1997). Experimental evidence supporting the disadvantageous effects, or fitness costs, of resistance factors has been growing (Foster et al., 1999). Although this study suggests that the resistance 
factor(s) on the third chromosome from the resistant line could have a disadvantageous effect on fitness measures (Table 3), this does not necessarily mean that all of the resistance factors have fitness disadvantages. There could be much variation in disadvantageous effects among resistance factors, and indeed, there are variations in fitness costs among resistance factors for organophosphate insecticides in the mosquito Culex pipiens L., from no effect to severe levels (Chevillon et al., 1997; Guillemaud et al., 1998; Berticat et al., 2002). Furthermore, the existence of fitness modifiers of resistant genotypes for an organophosphate, diazinon was demonstrated in the Australian sheep blowfly, Lucilia cuprina (Wiedemann) (McKenzie et al., 1982; McKenzie and Purvis, 1984; McKenzie and Game, 1987).

One of the major exceptions in this study is that the fitness of line RRR was not significantly different from the line SSS (Fig. 1), even though line RRR possessed all of the major chromosomes from the resistant line \#60910. In fact, significant positive contributions of the interactions among the resistant chromosomes to these fitness measures were detected (Table 3). Because the second chromosome from the resistant line, which did not have a significant effect on resistance to the three organophosphates (Miyo and Oguma, 2002), also has a significant negative effect on these fitness measures, there might be some epistatic interactions between the resistance factor(s) and other deleterious mutations involved. The Katsunuma population has been indeed demonstrated to maintain relatively high frequencies of fitnessrelated mutations, affecting traits such as viability (Watanabe and Oshima, 1970), fertility (Oshima and Watanabe, 1973; Watanabe and Oshima, 1973; Watanabe and Watanabe, 1973), and productivity (Watanabe and Ohnishi, 1975). However, we only analyzed chromosome-substituted lines derived from one resistant and one susceptible line. We thus need to separate the putative disadvantageous effect of the resistance factor(s) from those of other potentially deleterious mutations on the third chromosome, although a deleterious effect of the second chromosome from the resistant line has been excluded. To verify the generality of the obtained results, several other resistant and susceptible lines are necessary to be examined.

In conclusion, we compared the intrinsic rate of increase and other fitness measures among chromosomesubstituted lines between a resistant and a susceptible line. The results suggest that the resistance factor(s) on the third chromosome from the resistant line could have disadvantageous effects on fitness and tend to decrease in frequency within the Katsunuma natural population in the fall. However, other complicating effects, such as epistatic interactions between the resistance factor(s) and other deleterious mutations, may be involved in the dynamics of resistance factors within natural popula- tions. Further research is necessary to reveal the population genetic process of insecticide resistance in the real natural populations.

We would like to thank two anonymous reviewers for critical comments on the manuscript.

\section{REFERENCES}

Anderson, W. W., and Watanabe, T. K. (1974) Selection by fertility in Drosophila pseudoobscura. Genetics 77, 559-564.

Berticat, C., Boquien, G., Raymond, M., and Chevillon, C. (2002) Insecticide resistance genes induce a mating competition cost in Culex pipiens mosquitoes. Genet. Res. Camb. 79, $41-47$.

Caswell, H. (2001) Matrix population models, 2nd edn. Sinauer Associates, Sunderland, Massachusetts, USA.

Charlesworth, B. (1972) Selection in populations with overlapping generations. III. Conditions for genetic equilibrium. Theor. Popul. Biol. 3, 377-395.

Charlesworth, B. (1993) Evolutionary mechanisms of senescence. Genetica 91, 11-19.

Charlesworth, B. (1994) Evolution in age-structured populations, 2nd edn. Cambridge University Press, Cambridge, UK.

Charlesworth, B., and Hughes, K. A. (2000) The maintenance of genetic variation in life-history traits. In: Evolutionary Genetics: from Molecules to Morphology (eds.: R. S. Singh and C. B. Krimbas), pp. 369-392. Cambridge University Press, Cambridge, UK.

Chevillon, C., Bourguet, D., Rousset, F., Pasteur, N., and Raymond, M. (1997) Pleiotropy of adaptive changes in populations: comparisons among insecticide resistance genes in Culex pipiens. Genet. Res. Camb. 70, 195-204.

Crow, J. F. (1957) Genetics of insect resistance to chemicals. Ann. Rev. Entomol. 2, 227-246.

Daborn, P. J., Yen, J. L., Bogwitz, M. R., Goff, G. Le, Feil, E., Jeffers, S., Tijet, N., Perry, T., Heckel, D., Batterham, P., Feyereisen, R., Wilson, T. G., and ffrench-Constant, R. H. (2002) A single P450 allele associated with insecticide resistance in Drosophila. Science 297, 2253-2256.

Foster, S. P., Woodcock, C. M., Williamson, M. S., Devonshire, A. L., Denholm, I., and Thompson, R. (1999) Reduced alarm response by peach-potato aphids, Myzus persicae (Hemiptera: Aphididae), with knock-down resistance to insecticides $(k d r)$ may impose a fitness cost through increased vulnerability to natural enemies. Bull. Entomol. Res. 89, 133-138.

Guillemaud, T., Lenormand, T., Bourguet, D., Chevillon, C., Pasteur, N., and Raymond, M. (1998) Evolution of resistance in Culex pipiens: allele replacement and changing environment. Evolution 52, 443-453.

Hatano, Y., Inoue, Y., Watada, M., Akai, S. M., Watanabe, T. K., Yamamoto, M., Hirai, K., Takegawa, H., and Itoh, M. (1999) Further genetic studies on the Katsunuma population of Drosophila melanogaster. Genes Genet. Syst. 74, 219-225.

Houle, D., Hoffmaster, D. K., Assimacopoulos, S., and Charlesworth, B. (1992) The genomic mutation rate for fitness in Drosophila. Nature 359, 58-60.

Houle, D., Hughes, K. A., Assimacopoulos, S., and Charlesworth, B. (1997) The effects of spontaneous mutation on quantitative traits. II. Dominance of mutations with effects on lifehistory traits. Genet. Res. Camb. 70, 27-34.

Hughes, K. A. (1995) The inbreeding decline and average dominance of genes affecting male life-history characters in 
Drosophila melanogaster. Genet. Res. Camb. 65, 41-52.

Johnston, G. (2000) SAS software to fit the generalized linear model. http://www.sas.com/rnd/app/papers/genmod.

Lenski, R. E., and Service, P. M. (1982) The statistical analysis of population growth rates calculated from schedules of survivorship and fecundity. Ecology 63, 655-662.

Manly, B. F. J. (1985) The Statistics of Natural Selection on Animal Populations. Chapman and Hall, London, UK.

McGraw, J. B., and Caswell, H. (1996) Estimation of individual fitness from life history data. Am. Nat. 147, 47-64.

McKenzie, J. A., and Game, A. Y. (1987) Diazinon resistance in Lucilia cuprina; mapping of a fitness modifier. Heredity 59, 371-381.

McKenzie, J. A., and Purvis, A. (1984) Chromosomal localisation of fitness modifiers of diazinon resistance genotypes of $\mathrm{Luci}$ lia cuprina. Heredity 53, 625-634.

McKenzie, J. A., Whitten, M. J., and Adena, M. A. (1982) The effect of genetic background on the fitness of diazinon resistance genotypes of the Australian sheep blowfly, Lucilia cuprina. Heredity 49, 1-9.

Miyo, T., Akai, S., and Oguma, Y. (2000) Seasonal fluctuation in susceptibility to insecticides within natural populations of Drosophila melanogaster: empirical observations of fitness costs of insecticide resistance. Genes Genet. Syst. 75, 97104.

Miyo, T., Kono, Y., and Oguma, Y. (2002) Genetic basis of crossresistance to three organophosphate insecticides in Drosophila melanogaster (Diptera: Drosophilidae). J. Econ. Entomol. 95, 871-877.

Miyo, T., and Oguma, Y. (2002) Negative correlations between resistance to three organophosphate insecticides and productivity within a natural population of Drosophila melano- gaster (Diptera: Drosophilidae). J. Econ. Entomol. 95, 1229-1238.

Miyo, T., Takamori, H., Kono, Y., and Oguma, Y. (2001) Genetic variation and correlations among responses to five insecticides within natural populations of Drosophila melanogaster (Diptera: Drosophilidae). J. Econ. Entomol. 94, 223-232.

Oshima, C., and Watanabe, T. K. (1973) Fertility genes in natural populations of Drosophila melanogaster. I. Frequency, allelism and persistence of sterility genes. Genetics, 74, 351-361.

Roush, R. T., and McKenzie, J. A. (1987) Ecological genetics of insecticide and acaricide resistance. Annu. Rev. Eotomol. 32, 361-380.

SAS Institute. (1985) SAS User's Guide: Statistics. SAS Institute, Cary, NC.

Simmons, M. J., and Crow, J. F. (1977) Mutations affecting fitness in Drosophila populations. Ann. Rev. Genet. 11, 4978.

Watanabe, T. K., and Ohnishi, S. (1975) Genes affecting productivity in natural populations of Drosophila melanogaster. Genetics 80, 807-819.

Watanabe, T. K., and Oshima, C. (1970) Persistence of lethal genes in Japanese natural populations of Drosophila melanogaster. Genetics 64, 93-106.

Watanabe, T. K., and Oshima, C. (1973) Fertility genes in natural populations of Drosophila melanogaster II. Correlations between productivity and viability. Jpn. J. Genet. 48, 337347.

Watanabe, T. K., and Watanabe, T. (1973) Fertility genes in natural populations of Drosophila melanogaster. III. Superiority of inversion heterozygotes. Evolution 27, 468-475. 